\title{
In-hospital experience with insulin degludec (IDeg)
}

\author{
Maria Gabriela Pedigoni Bulisani, Maria Fernanda Ozorio de Almeida*, Camila Miranda Abdon, \\ Ana Cláudia Souza Moreno, Marília Izar Helfenstein Fonseca, Paulo Roberto Rizzo Genestreti \\ From 20th Brazilian Diabetes Society Congress \\ Porto Alegre, Brazil. 11-18 November 2015
}

\section{Background}

Glycemic control is critical for in-patients and dysglycemia is associated with worse prognosis and higher mortality. Insulin therapy is considered the best treatment on this scenario and basal insulin analogues, such as insulin glargine (IGlar) and IDeg, could be useful options, however there are no studies comparing IGlar and IDeg evaluating glucose variability (GV) in hospitalized patients, neither the transition between them.

\section{Objective}

To present a case series describing the efficacy and safety of IDeg in-patientes with diabetes using GV and rate of hypoglycemia when compared with IGlar.

\section{Materials and methods}

Retrospective analysis of blood glucose obtained with point-of-care testing of 10 diabetic patients admitted at Bandeirantes Hospital, Sao Paulo, between October 2014 and April 2015, previously treated with IGlar for a minimum of 7 days and switched to IDeg for at least 7 days more during hospitalization. Parameters studied included GV, standard deviation (SD), coefficient of variation $(\mathrm{CV})$ and mean glucose levels, obtained from software PXP Abbott. Hypoglycemia was defined as blood glucose (BG) $<70 \mathrm{mg} / \mathrm{dL}$ and it was severe if $\mathrm{BG}<40 \mathrm{mg} /$ $\mathrm{dL}$. Basal insulin dose was compared on the last day of both, after achievement of steady state.

\section{Results}

3/10 patients had type 1 diabetes mellitus (T1D) for over 10 yrs., previous treatment with insulin therapy in a basal-bolus regimen. Average age was 46 yrs. and mean $\mathrm{HbA} 1 \mathrm{c}$ was $9 \%$ and no benefit could be noticed. $7 / 10$ patients had type 2 diabetes mellitus (T2D), with duration of $>10$ yrs., the majority had previous insulin treatment. Mean age was 70 yrs. and mean HbA1c was 9,7\%. All T2D patients maintained CV, and $57 \%$ had a reduction in SD, improving GV. Basal insulin dose with IDeg was lower at discharge as compared to IGlar in T2D. Severe hypoglycemia events were diminished after switching (see Figure 1).

\section{Conclusions}

In this report, GV was lower in T2D patients treated with IDeg as compared to IGlar, although the same could not be seen in T1D, perhaps due to the small number of patients included. More studies in this population are needed to confirm this hypothesis and continuous glucose monitoring should be preferred. IDeg proved to be a safe and effective alternative at hospital and might improve GV. This would be better due to its predictability of effect and low rate of hypoglycemia, important among hospitalized patients.

Published: 11 November 2015

doi:10.1186/1758-5996-7-S1-A90

Cite this article as: Bulisani et al:: In-hospital experience with insulin degludec (IDeg). Diabetology \& Metabolic Syndrome 2015 7(Suppl 1):A90.

* Correspondence: nandaozorio@hotmail.com

Santa Casa, São Paulo, Brazil

(c) 2015 Bulisani et al. This is an Open Access article distributed under the terms of the Creative Commons Attribution License (http:// creativecommons.org/licenses/by/4.0), which permits unrestricted use, distribution, and reproduction in any medium, provided the original work is properly cited. The Creative Commons Public Domain Dedication waiver (http://creativecommons.org/publicdomain/ zero/1.0/) applies to the data made available in this article, unless otherwise stated. 


\begin{tabular}{|c|c|c|c|c|c|c|c|c|c|c|}
\hline & Sex & Age (y) & $\begin{array}{l}\text { Typ } \\
\text { e of } \\
\text { DM }\end{array}$ & $\begin{array}{l}\text { Cause for in- } \\
\text { hospital } \\
\text { admission }\end{array}$ & $\begin{array}{c}\text { Previous } \\
\text { DM } \\
\text { Treatment }\end{array}$ & $\begin{array}{c}\text { Duratio } \\
\mathrm{n} \text { of DM } \\
\text { (y) }\end{array}$ & $\begin{array}{l}\text { CV IGlar } \\
\text { vs IDeg }\end{array}$ & $\begin{array}{c}\text { SD } \\
\text { IGlar } \\
\text { vs } \\
\text { IDeg }\end{array}$ & $\begin{array}{c}\text { Severe } \\
\text { hypo } \\
\text { Glar vs } \\
\text { IDeg }\end{array}$ & $\begin{array}{c}\text { Basal insulin } \\
\text { dose IGlar } \\
\text { vs IDeg at } \\
\text { discharge }\end{array}$ \\
\hline 1 & $\mathrm{~F}$ & 89 & 2 & Clinical & $\begin{array}{c}\text { Basal } \\
\text { insulin + } \\
\text { OAD }\end{array}$ & $<5$ & $\begin{array}{c}29 \% \text { vs } \\
24 \%\end{array}$ & $\begin{array}{c}49,11 \\
\text { vs } \\
36,39\end{array}$ & 0 vs 0 & 20 vs 20 \\
\hline 2 & $M$ & 76 & 2 & Clinical & $\begin{array}{c}\text { Basal } \\
\text { insulin + } \\
\text { OAD }\end{array}$ & $>10$ & $\begin{array}{c}32 \% \text { vs } \\
44 \%\end{array}$ & $\begin{array}{c}48,39 \\
\text { vs } \\
76,83\end{array}$ & 0 vs 0 & 40 vs 28 \\
\hline 3 & $M$ & 82 & 2 & Clinical & OAD & $>10$ & $\begin{array}{c}27 \% \text { vs } \\
14 \%\end{array}$ & $\begin{array}{c}4,49 \\
\text { vs } \\
26,54\end{array}$ & 0 vs 0 & 10 vs 12 \\
\hline 4 & $M$ & 58 & 2 & Clinical & $\begin{array}{c}\text { Basal- } \\
\text { bolus + } \\
\text { OAD }\end{array}$ & $>10$ & $\begin{array}{c}36 \% \text { vs } \\
29 \%\end{array}$ & $\begin{array}{c}49,69 \\
\text { vs } \\
36,59\end{array}$ & 0 vs 0 & 56 vs 52 \\
\hline 5 & $\mathrm{~F}$ & 57 & 2 & Clinical & None & $<5$ & $\begin{array}{c}46 \% \text { vs } \\
31 \%\end{array}$ & $\begin{array}{c}70,11 \\
\text { vs } \\
44,98\end{array}$ & 1 vs 0 & 14 vs 12 \\
\hline 6 & $M$ & 47 & 1 & Clinical & $\begin{array}{l}\text { Basal- } \\
\text { bolus }\end{array}$ & $>10$ & $\begin{array}{c}41 \% \text { vs } \\
31 \%\end{array}$ & $\begin{array}{c}67,35 \\
\text { vs } \\
60,0\end{array}$ & 0 vs 0 & 20 vs 14 \\
\hline 7 & $\mathrm{~F}$ & 80 & 2 & Clinical & $\begin{array}{c}\text { Basal- } \\
\text { bolus + } \\
\text { OAD }\end{array}$ & $<5$ & $\begin{array}{c}36 \% \text { vs } \\
40 \%\end{array}$ & $\begin{array}{c}66,54 \\
\text { vs } \\
91,32\end{array}$ & 1 vs 0 & 14 vs 22 \\
\hline 8 & M & 54 & 2 & Clinical & $\begin{array}{c}\text { Basal } \\
\text { insulin + } \\
\text { OAD }\end{array}$ & $>10$ & $\begin{array}{c}37 \% \text { vs } \\
59 \%\end{array}$ & $\begin{array}{l}62,67 \\
\text { vs } 124\end{array}$ & 0 vs 0 & 50 vs 46 \\
\hline 9 & $\mathrm{~F}$ & 39 & 1 & Clinical & $\begin{array}{l}\text { Basal- } \\
\text { bolus }\end{array}$ & $>10$ & $\begin{array}{c}63 \% \text { vs } \\
59 \%\end{array}$ & $\begin{array}{c}91,62 \\
\text { vs } \\
121,8\end{array}$ & 3 vs 1 & 20 vs 18 \\
\hline 10 & $\mathrm{~F}$ & 54 & 1 & Surgical & $\begin{array}{c}\text { Basal - } \\
\text { bolus }\end{array}$ & $>10$ & $\begin{array}{c}32 \% \text { vs } \\
56 \%\end{array}$ & $\begin{array}{l}53,18 \\
\text { vs } 99\end{array}$ & 0 vs 0 & 20 vs 20 \\
\hline \multicolumn{11}{|c|}{$\begin{array}{l}\text { Y: years; M: male; F: female; DM: diabetes mellitus; OAD: oral antidiabetic medication; CV: coefficient of } \\
\text { variation; SD: standard deviation; hypo: htpoglycemia; vs: versus. }\end{array}$} \\
\hline
\end{tabular}

